BNL- 112646-2016--JA

\title{
Excitonic Lasing in Solution-Processed Subwavelength Nanosphere Assemblies
}

\author{
Kannatassen Appavoo, Xiaoze Liu, \\ Vinod Menon and Matthew Y. Sfeir
}

Submitted to Nano Letters

March 2016

Center for Functional Nanomaterials

Brookhaven National Laboratory

\author{
U.S. Department of Energy \\ USDOE Office of Science (SC), \\ Basic Energy Sciences (SC-22)
}

\footnotetext{
Notice: This manuscript has been authored by employees of Brookhaven Science Associates, LLC under Contract No. DE- SC0012704 with the U.S. Department of Energy. The publisher by accepting the manuscript for publication acknowledges that the United States Government retains a non-exclusive, paid-up, irrevocable, world-wide license to publish or reproduce the published form of this manuscript, or allow others to do so, for United States Government purposes.
} 


\section{DISCLAIMER}

This report was prepared as an account of work sponsored by an agency of the United States Government. Neither the United States Government nor any agency thereof, nor any of their employees, nor any of their contractors, subcontractors, or their employees, makes any warranty, express or implied, or assumes any legal liability or responsibility for the accuracy, completeness, or any third party's use or the results of such use of any information, apparatus, product, or process disclosed, or represents that its use would not infringe privately owned rights. Reference herein to any specific commercial product, process, or service by trade name, trademark, manufacturer, or otherwise, does not necessarily constitute or imply its endorsement, recommendation, or favoring by the United States Government or any agency thereof or its contractors or subcontractors. The views and opinions of authors expressed herein do not necessarily state or reflect those of the United States Government or any agency thereof. 


\title{
Excitonic Lasing in Solution-Processed
}

\section{Subwavelength Nanosphere Assemblies}

\author{
Kannatassen Appavoo, ${ }^{1}$ Xiaoze Liu, ${ }^{2}$ Vinod Menon ${ }^{2}$ and Matthew Y. Sfeir ${ }^{1 *}$ \\ ${ }^{1}$ Center for Functional Nanomaterials, Brookhaven National Laboratory, Upton, NY 11973 \\ ${ }^{2}$ Department of Physics, City College of New York, NY, 10031
}

Keywords: cavity-free, room-temperature lasing, solution-processed film, ultrafast dynamics, electronphonon coupling

Lasing in solution-processed nanomaterials has gained significant interest because of the potential for low-cost integrated photonic devices. Still, a key challenge is to utilize a comprehensive knowledge of the system's spectral and temporal dynamics to design low-threshold lasing devices. Here we demonstrate intrinsic lasing at low-threshold in an ultrathin film of coupled, highly crystalline nanospheres, with overall thickness on the order of $\sim \lambda / 4$. The cavity-free geometry consists of $\sim 35 \mathrm{~nm}$ zinc oxide nanospheres that collectively localize the in-plane emissive light fields while minimizing scattering losses, resulting in excitonic lasing with fluence thresholds at least an order of magnitude lower than previous UV-blue random and quantum-dot lasers $\left(<75 \mu \mathrm{J} / \mathrm{cm}^{2}\right)$. Fluence-dependent effects, as quantified by sub-picosecond transient spectroscopy, highlight the role of phonon-mediated processes in excitonic lasing. Sub-picosecond evolution of distinct lasing modes, together with 3D electromagnetic simulations, indicate a random lasing process - in violation of the commonly cited criteria of strong scattering from individual nanostructures and an optically thick sample. Subsequently, an electron-hole plasma mechanism is observed with increased fluence. These results suggest that coupled nanostructures with high crystallinity, fabricated by low-cost solution-processing methods, can function as viable building blocks for high-performance optoelectronics devices. 
For integrated photonic applications, it is necessary to develop low cost, high-efficiency light amplification processes. While various strategies have been explored over the last decade ${ }^{1-3}$, two distinct schemes have been investigated for disordered media - amplified spontaneous emission in thin films of nanometer-sized quantum dot nanostructures (QD-ASE) and random lasing in larger, micron-sized structures. Each strategy has critical inherent drawbacks that have so far limited their practical use. In QD-ASE devices, weakly scattering nanometer-sized particles allow for the long optical path lengths necessary to achieve optical gain. As a result, the incoherent emission profile is derived solely from the gain curve of the medium, which is tunable via the size dependent optical properties of quantum dots. However, relatively large critical volumes are required for light amplification in these systems and their efficiency is highly sensitive to the initial excitonic states that are excited and their relaxation dynamics. ${ }^{4-7}$ As a result, complex band engineering strategies, for example, core/shell nanostructures, are necessary to reduce losses via non-radiative Auger recombination and reabsorption. ${ }^{1,-11}$ In contrast, in random lasers, constructive light interference via multiple scattering leads to the evolution of distinct modes in the lasing spectrum. Here, materials are typically chosen to provide both scattering and gain functionality, either by mixing non-resonant scatterers with a gain medium such as a laser dye ${ }^{12-14}$ or by using bifunctional materials that provide both. ${ }^{15-17}$ While considerable work has been done to tune the particle size to optimize resonant scattering at the band-edge emission wavelength, ${ }^{18-20}$ losses remain high, leading to lasing thresholds in the few $\mathrm{mJ} / \mathrm{cm}^{2}$ range. ${ }^{18}$ As a result of these challenges, current QD-ASE and random lasing schemes do not offer the ideal device characteristics of both small critical dimensions and low lasing threshold. 
In this Letter, we demonstrate the emergence of short-lived collective emission modes through a low threshold, excitonic lasing process in sub-wavelength thin films of $\mathrm{ZnO}$ nanospheres (average diameter of $\sim 35 \mathrm{~nm}$ ) with weak individual scattering properties. In contrast to previous studies that have focused mainly on maximizing the scattering efficiency of individual nanostructures at the lasing wavelength, with predicted optimal sizes of $\sim 260 \mathrm{~nm},{ }^{18-20}$ here we show that efficient, low-threshold lasing is achieved by using much smaller, highly-crystalline nanostructures with combined effects of increasing many-body interactions (i.e. carrier-carrier, carrier-phonon). The gain is enhanced by the accumulated in-plane emission scattering while the loss rate is minimized by reducing "voids" in the nanostructured film when the size of the nanospheres is decreased. This strategy allows us to achieve lasing even in films with thickness of $\sim \lambda_{\text {emission }} / 4$. Overall, this system achieves an order of magnitude reduction in the fluence threshold in films a few orders of magnitude thinner than previously reported ${ }^{14,17-18,21}$. This nanostructured lasing medium has characteristics of both QD-ASE and random lasing in so far that distinct modes are seen transiently in emission, while the low lasing thresholds $(<75$ $\mu \mathrm{J} / \mathrm{cm}^{2}$ ) enable the excitonic lasing condition, i.e., lasing at photoexcited carrier densities below the Mott transition. Using an ultrafast transient emission method, we quantify the effect of fluence on these many-body interactions that lead to excitonic lasing, on the spectral and temporal dynamical evolution of the various lasing modes, and on the build-up time of the lasing process.

To fabricate the sub-wavelength random lasers, we assemble highly crystalline zinc oxide nanospheres $^{22}$, with high refractive index $(\mathrm{n}=2.3)$ and strong photoluminescence in the $\mathrm{UV}$, into thin films via solution processing methods (Figure 1a, see also Figure S1 in Supplementary Information). We observed emission line narrowing and threshold behavior at $\sim 65 \mu \mathrm{J} / \mathrm{cm}^{2}$, above 
which the emission intensity changes by 3 orders of magnitude $(N S F$, Fig. 1b,c), indicative of a lasing process. Threshold behavior is always observed in films with thickness greater than 100 $\mathrm{nm}$ as measured by scanning electron microscopy and ellipsometry, with values for average thickness that are roughly quantized by the number of $35 \mathrm{~nm}$ diameter nanostructures in the outof-plane direction $(\sim 105 \mathrm{~nm}, 140 \mathrm{~nm}$, and $160 \mathrm{~nm}$ for 3, 4, and 5, respectively). Threshold behavior was observed over the full range of pump-spot sizes were able to access, from $2.2 \mu \mathrm{m}-$ $1 \mathrm{~mm}$ (Figure S2).

To confirm that these emission features are derived from a collective process, we also show that no line narrowing or threshold behavior is observed in (i) individual $\mathrm{ZnO}$ nanospheres $(d-N S$ for NS dispersed in solution, gray curve in Fig. 1b), (ii) samples that had thicknesses less than a $100 \mathrm{~nm}$ (i.e. < 3 nanospheres thick), or (iii) in any nanosphere-films in which a rapid, hightemperature annealing process was employed ( $a-N S F$, orange curve in Fig. 1b,c). Furthermore, we observe drastic changes in the temporal behavior of the emission above threshold in addition to changes in the intensity and emission lineshape. Using an ultrafast transient emission technique (Methods, Figure S3), we observe a picosecond time scale emission process that is at least an order of magnitude faster than in an isolated nanostructure or films excited under nonlasing conditions (Fig. 1d). Together, these results demonstrate an emission process that is highly sensitive to both geometric (density, size, film morphology) and electronic considerations; while a high density of nanospheres is an imperative requisite for gain, fusing of the nanospheres during this rapid annealing process changes the scattering profile and introduces interfacial trap states that inhibit the lasing action. 

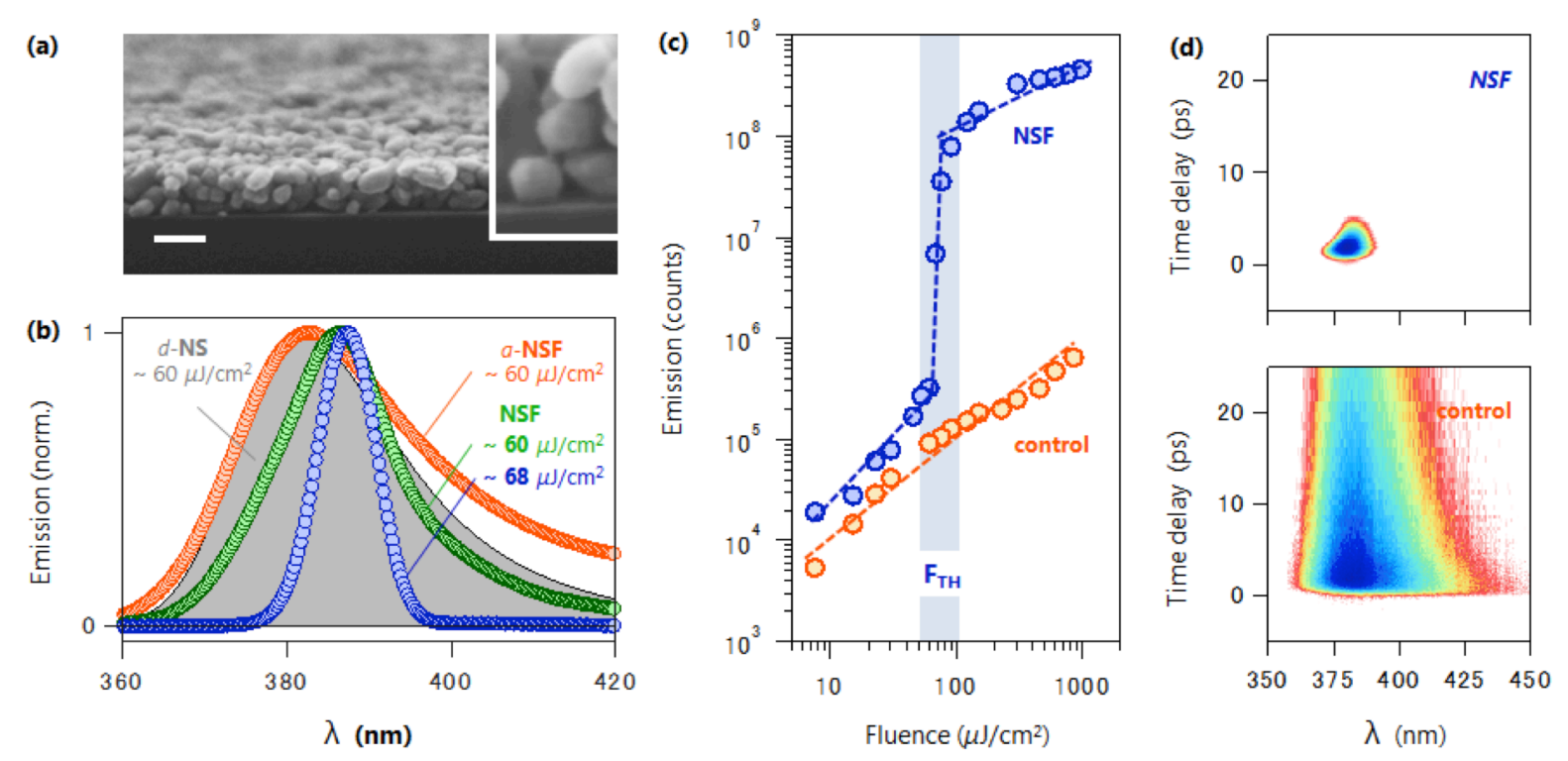

Figure 1. Coupling Effect of Nanostructures on Emission Intensity. (a) Scanning electron micrographs of thin-film coupled nanostructures (NSF) exhibiting lasing. Scale bar is $100 \mathrm{~nm}$ and nanospheres are $\sim 35 \mathrm{~nm}$ in diameter. (b) Normalized emission spectra for pristine NSF near threshold $\left(60 \mu \mathrm{J} / \mathrm{cm}^{2}\right.$, green) and $68 \mu \mathrm{J} / \mathrm{cm}^{2}$, blue), along with one annealed for a short duration ( $a$-NSF, orange) and when nanospheres are dispersed in ethanol ( $d$-NS, shaded grey). Note that the broader linewidth for the anneal sample is due to emission from interfacial defect states that contribute to the lower energy tail of the band-edge emission. (c) Peak emission intensity at the band-edge as a function of pump fluence for a pristine film of nanospheres (NSF, blue) and one annealed for a short duration ( $a$-NSF, blue). (d) $2 \mathrm{D}$ time-resolved emission measurement of coupled-NSs thin-film $\left(100 \mu \mathrm{J} / \mathrm{cm}^{2}\right)$ and representative control sample (here, individual nanospheres dispersed in ethanol). Similar 2D plots were obtained for the coupled-NSs when excited with lower than threshold fluence and for the rapidly annealed sample.

While spectral line narrowing, threshold behavior, and fast emission all are highly suggestive of a lasing process, the criteria for proving random lasing necessitate a demonstration of optical 
gain. To confirm optical gain and understand the gain temporal profile, we use the linear and transient optical absorption spectra. Briefly, for transient absorption measurements, a $280 \mathrm{~nm}$ ultrafast laser pump pulse ( $100 \mathrm{fs})$ create a density of electron-hole pairs above the optical bandgap, while a weak broadband probe pulse, time-delayed with respect to the pump, monitors the relaxation dynamics of the excited carriers returning to the ground state ${ }^{7,23}$. To highlight the nonlinear evolution of the system, the raw transient absorption data is processed so that the linear absorption of the sample (scattering corrected) is added to each transient spectrum. From this treatment, optical gain can be readily visualized as it corresponds to signals where the OD + $\Delta \mathrm{OD}$ value is negative ${ }^{6,24}$. A representative $2 \mathrm{D}$ data set, displaying $\mathrm{OD}+\Delta \mathrm{OD}$ as a function of time and probe wavelength, is shown in Fig. 2a for a pump fluence $\left(100 \mu \mathrm{J} / \mathrm{cm}^{2}\right)$ above the lasing threshold. The $\Delta \mathrm{A}$ scale is set to highlight the negative signal (optical gain) that develops in the region between $380-420 \mathrm{~nm}$. As in the steady-state emission measurements (Fig. 1C), the optical gain signal is nonlinear in the fluence and exhibits a threshold behavior. This can be readily seen in Fig. 2 b, which shows the transient spectrum at a fixed time ( 2 ps after excitation) as a function of pump fluence, where the nonlinear component emerges at pump fluences around $60-70 \mu \mathrm{J} / \mathrm{cm}^{2}(\mathrm{OD}+\mathrm{mOD}<0)$. A comparison of the linear and transient signals at few critical time delays is shown in Figure 2c. The gain lifetime lasts for less than 10 ps though a small transient signal persists for longer times. 

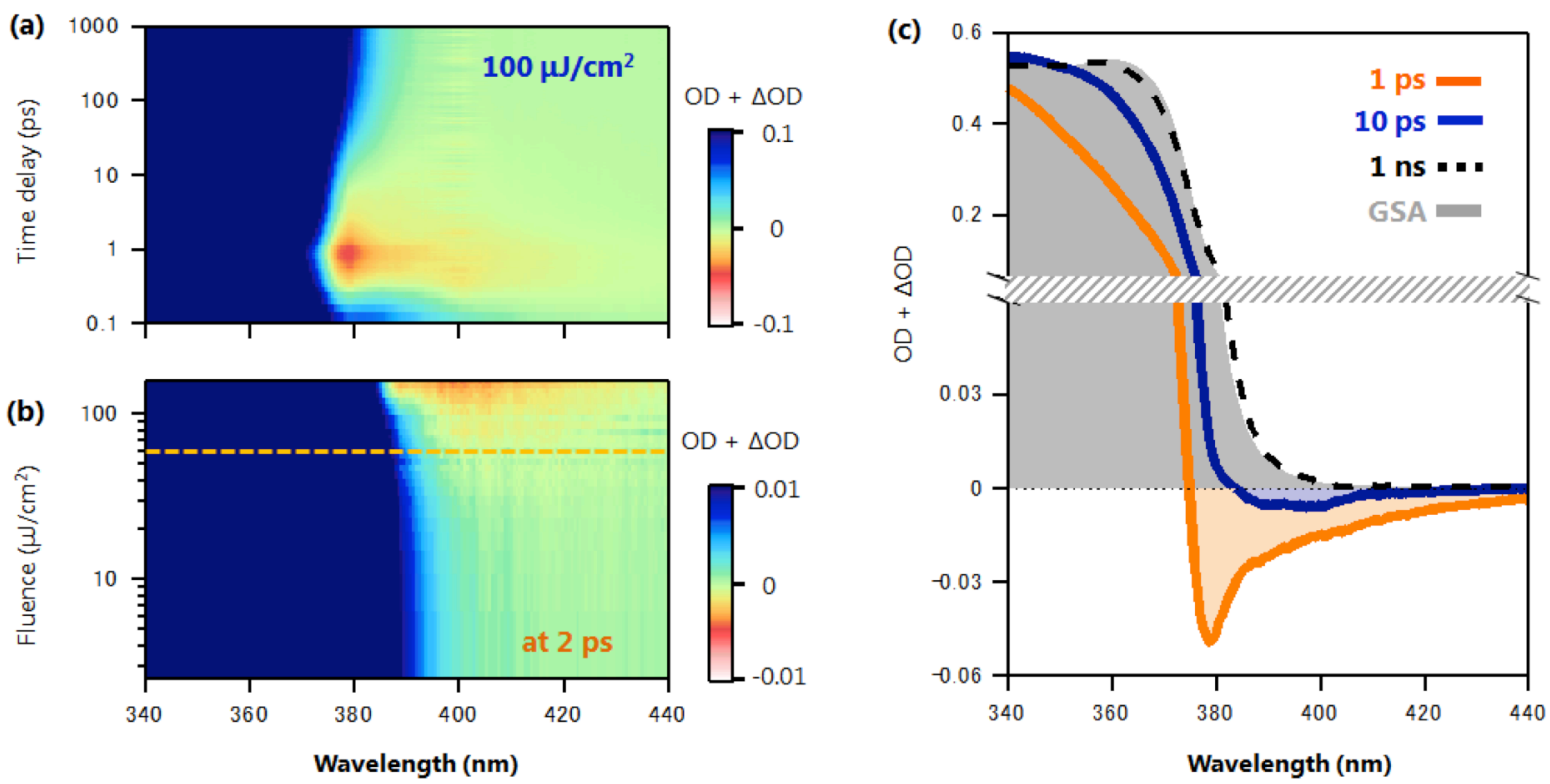

Figure 2. Optical Gain in a Coupled Nanosphere Film. (a) 2D-time resolved map of the optical gain, obtained by summing the linear and transient optical absorption. (b) Optical gain $(\mathrm{OD}+\Delta \mathrm{OD})$ at 2 ps after photoexcitation, plotted as a function of pump fluence. Yellow line is only a guide to the eye and represents the region near the fluence threshold. (c) Spectral slices of the optical gain map in (a) at 1 ps, $10 \mathrm{ps}$, and $1 \mathrm{~ns}$, along with the ground-state linear absorption.

Furthermore, we identify discrete lasing modes in the absence of an external optical cavity, the critical signature of a random lasing mechanism. This is apparent in the slight differences which we observe in the lasing spectra as a function of both sample position and in time (Figure S4). Furthermore, using a high sensitivity transient emission technique based on optical Kerr gating (Figure S3), described previously, we are able to resolve the temporal dynamics of individual modes with $\sim 200$ fs time resolution. ${ }^{25}$ By examining spectral slices at discrete times after pumping in a $140 \mathrm{~nm}$ thick $\mathrm{ZnO}-\mathrm{NSs}$ film (Figure S5), we observe that lasing modes are transiently populated as the system evolves. Since they evolve with a distinct time domain behavior, we can clearly see that the individual modes have a linewidth that is narrower than the 
time-averaged value. Taken together, these features establish that a random lasing process is occurring. The number of lasing modes and the overall bandwidth increases dramatically with increasing fluence, and on average, the red-shifted modes appear at later times that those on the blue edge of the spectrum. This time delay associated with the red-shifted modes is consistent a shift away from the maximum of the gain curve for $\mathrm{ZnO}$ along with decreased scattering efficiency. At higher fluences $\left(>300 \mu \mathrm{J} / \mathrm{cm}^{2}\right)$, more complex spectral dynamics are observed. We note that narrowing of the linewidth to less than a nanometer is not a necessary signature of random lasing. The observation of relatively broad lasing modes (low $\mathrm{Q}$ factor) is consistent with their ultrafast (sub-ps) lifetime. Also, using small nanospheres with a narrow size distribution provides a large number of optimum optical path lengths within the gain bandwidth.

In addition to understanding the mechanism that triggers lasing in those sub-wavelength assemblies of weakly scattering nanospheres, the ultrafast transient emission measurements allow us to understand differences in the lasing behavior under low and high fluence pump conditions. Below the lasing threshold $\left(29 \mu \mathrm{J} / \mathrm{cm}^{2}\right)$, relatively static emission dynamics are seen with a lifetime of $\sim 37 \mathrm{ps}$, similar to isolated nanostructures (Figure 3a). Near threshold $(\sim 60$ $\mu \mathrm{J} / \mathrm{cm}^{2}$ ), the first sign of lasing is apparent, with "spikes" of intense emission occurring at few100 fs intervals (see arrows in Figure 3b, and Figure 4a). These pronounced transient oscillations, or laser spiking, are a direct signature of the scattering mechanism that causes population inversion ${ }^{13,26}$ and a direct consequence of the sample trying to reduce the dwell time of the photons in the medium inversion..$^{27}$ 

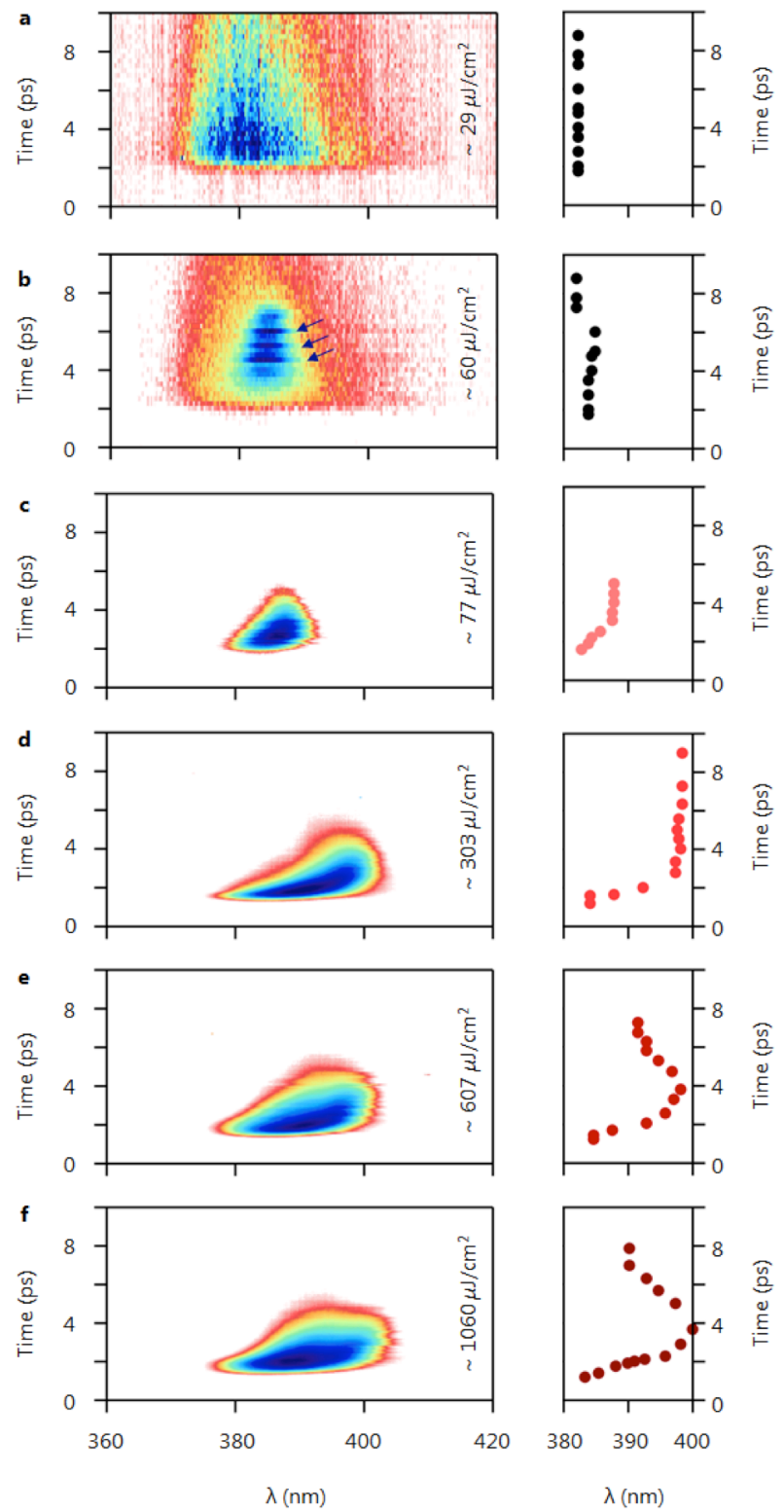

Figure 3. Emission Dynamics at Low and High Fluence. Evolution of the emission spectrum for the pristine-CNF as optical pump fluence is gradually increased from (a) $29 \mu \mathrm{J} / \mathrm{cm}^{2}$ to (f) $1060 \mu \mathrm{J} / \mathrm{cm}^{2}$. The $60 \mu \mathrm{J} / \mathrm{cm}^{2}$ pump fluence data is characteristic of the near-lasing threshold behavior and exhibitd both below and above threshold spectrotemporal features. Figures on the right side track the peak emission intensity with time. Above, $300 \mu \mathrm{J} / \mathrm{cm}^{2}$ signatures the transition from exciton to electron hole plasma lasing can be seen from the dynamics shifting of the emission maxima. Note that emission dynamics in figures (a-b) and (c-f) have been gated using the optical Kerr effect in benzene and glass respectively, with a temporal resolution of $\sim 530$ fs and $\sim 220$ fs. 

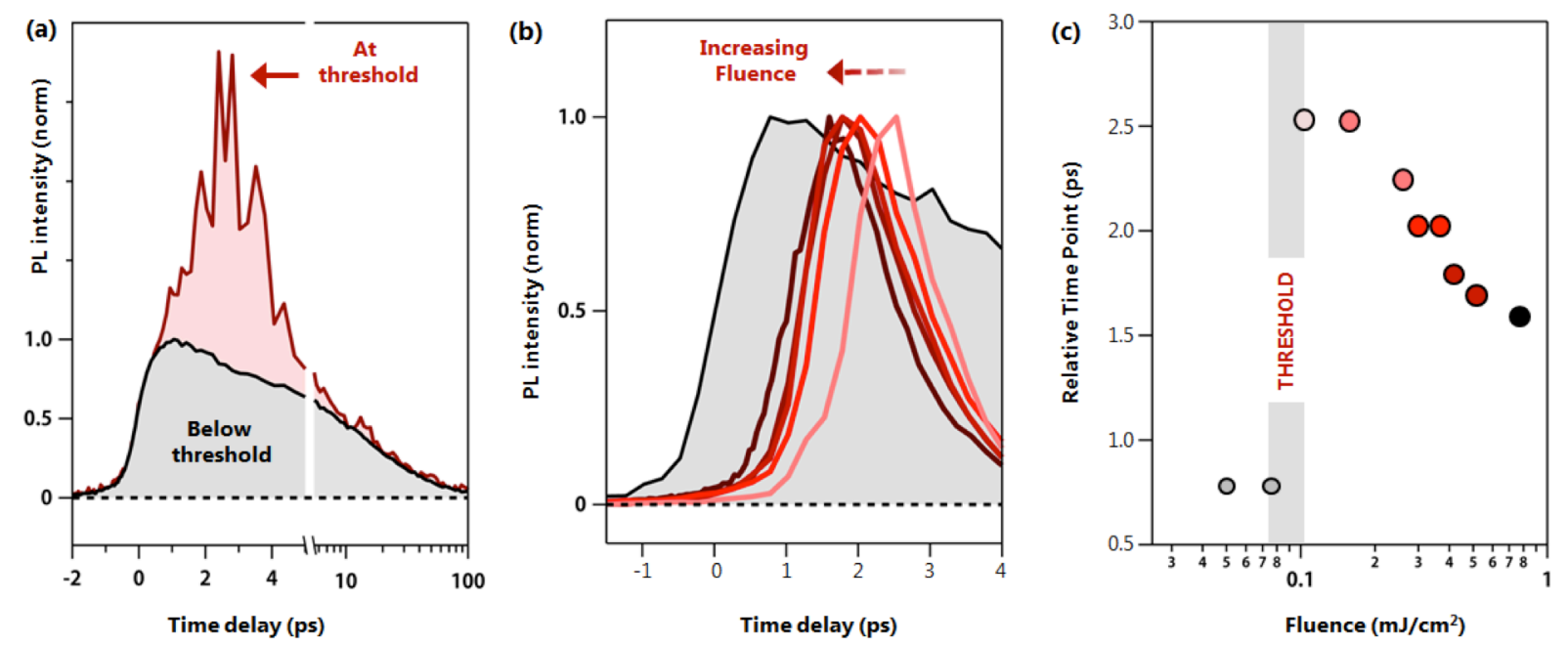

Figure 4. Fluence-Dependent Buildup Time on Lasing. (a) Kinetic traces at band-edge emission of thin-film coupled nanospheres below and at near-lasing threshold. At threshold, lasing instabilities can be seen superimposed on the normal fluorescence decay during the first few ps. (b) Kinetics traces for various pump fluences above lasing threshold and (c) the time delay of the emission maximum relative to the pump pulse arrival time as a function of pump fluence.

Under high fluence pumping, the complex lasing dynamics can be explained by the overlapping, ultrafast emission modes and the crossover from excitonic to electron-hole plasma lasing behavior. Just above threshold, the system is in the exciton lasing regime since the optical pump fluence corresponds to carrier densities of $\sim 1.5 \times 10^{18} \mathrm{~cm}^{-3}$, much lower than the Mott threshold value $\left(n_{M}=k_{B} T / 2 a_{o}^{3} E_{\text {exc }} \sim 3.7 \times 10^{19} \mathrm{~cm}^{-3}\right.$; where $a_{o}$ is the Bohr's radius of $18 \AA$ and $E_{\text {exc }}$ is the exciton binding energy of $\left.60 \mathrm{meV}\right) .{ }^{15}$ The spectral dynamics reinforce this conclusion, as an immediate redshift corresponding to P-band exciton scattering is seen in Figure $4 c .^{28-29}$ Although such spectral shifts have been reported in steady-state experiments on $\mathrm{ZnO}$ microcavities $^{30}$ and nanowires ${ }^{31}$, here the dynamic exciton-exciton scattering process is monitored in real time. With increasingly higher fluences, the initial exciton scattering 
mechanism ${ }^{32-34}$ is quickly subdued by the formation of an electron-hole plasma (EHP). The EHP signature is a drastic red-shift and broadening in the emission at early times followed by a dynamic blue shift as carriers decay to the ground state and the density drops below the plasma threshold ${ }^{35}$. This behavior is clearly seen by plotting the maxima of the lasing emission wavelength as a function of time (right panels of Figure 3). At higher fluences (Figure 3d-f), the emission maximum is initially red-shifted by $10 \mathrm{~nm}$ compared to $3 \mathrm{c}$, and exhibits a dynamic recovery of excitonic lasing at $390 \mathrm{~nm}$ after a few picoseconds due to the decay of excess carrier density and a return to the excitonic regime.

The dependence of the lasing buildup time on the excitonic density, i.e. pump fluence, suggest that electron-phonon coupling is important for achieving low-threshold lasing. After precisely determining time zero based on the arrival time of the excitation pulse, we plot the kinetics of the system at the emission maximum (Figure 4a,b) and the buildup time (Figure 4c) for different pump fluences. Notably, the maximum delay of $\sim 2.5$ ps occurs precisely at the lasing fluence threshold. The change in buildup time highlights the complicated interaction between excited carriers and the phonon modes that exist in the gain material, and which have been shown to be crucial for lasing in II-VI semiconductors ${ }^{36}$ in the low fluence regime ${ }^{37}$. Moreover, this delay highlights the importance of phonon modes in distributing excitons within the continuum states ${ }^{31}$, ${ }^{38-39}$, thus reducing intraband non-radiative exciton-exciton scattering and ultimately fulfilling the population inversion that initially triggers lasing. With an increased density of excited carriers, additional carrier-carrier scattering into the continuum states ${ }^{40}$ occurs and the temporal delay in lasing is reduced.

Additional insight into the mechanism of exciton random lasing in the sub-wavelength limit is obtained using full-field 3D electromagnetic simulations (see details in Methods section), which 
confirm that lasing is seeded from the collective scattering of the nanospheres. These simulations explored the effect of film morphology and disorder on the ability to localize and enhance the electromagnetic field over the relevant spectral range $(350-450 \mathrm{~nm})$. In Figure 5, we plot the electric field enhancement, $|E|^{2}$, at the emission peak $(\sim 403 \mathrm{~nm})$ in a $140 \mathrm{~nm}$ thin film composed of unstructured $\mathrm{ZnO}$, a close-packed film composed of $\mathrm{ZnO}$ nanospheres with diameter of 40 $\mathrm{nm}$, and a film of randomly arranged $\mathrm{ZnO}$ nanospheres with a distribution of diameters between 40 and $50 \mathrm{~nm}$. Significant enhancement near the $\mathrm{ZnO}$ bandedge emission occurs only for the film composed of randomly packed, heterogeneous $\mathrm{ZnO}$ nanospheres. Identically thick films of unstructured and well-aligned close-packed $\mathrm{ZnO}$ do not show a similar dramatic increase in scattering at any relevant wavelength. These results show that the randomly arranged nanospheres are required to confine the light fields within the film and to consequently increase the in-plane scattering needed to achieve lasing. 

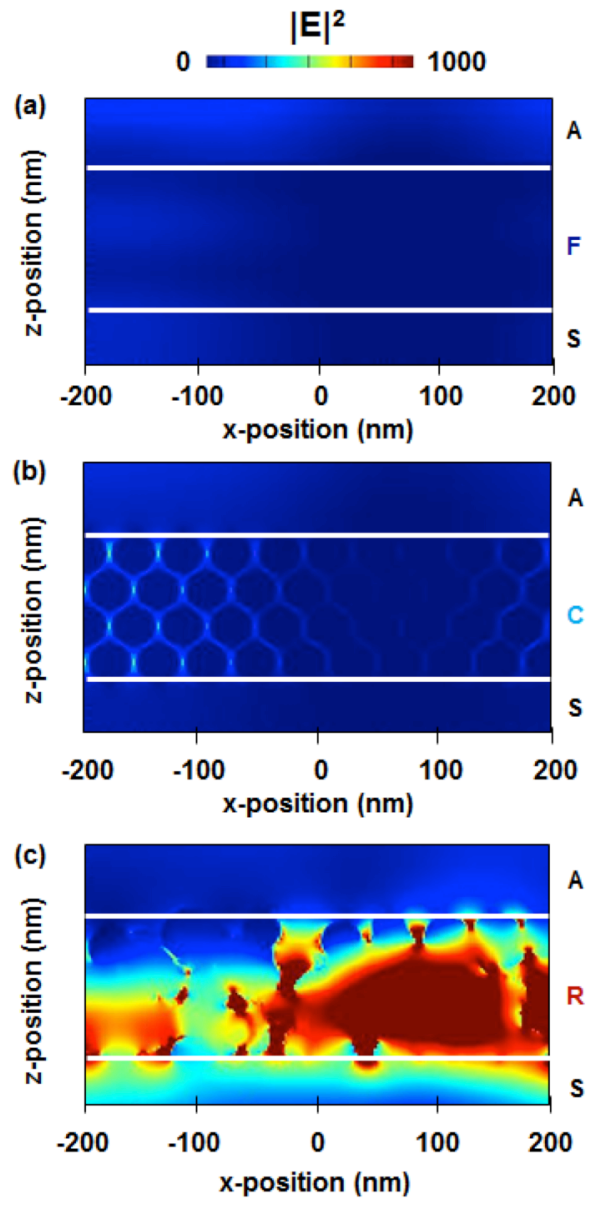

Figure 5. Near-Field Electromagnetic Distributions of Various ZnO Thin-Film Configurations. XZ-plane comparing the $|E|^{2}$ at the emission peak $(\sim 403 \mathrm{~nm})$ for (a) flat, unstructured thin-film, (b) close-packed film composed of $\mathrm{ZnO}$ nanospheres with diameter of $40 \mathrm{~nm}$ and (c) randomly arranged $\mathrm{ZnO}$ nanospheres with a uniform size distribution, ranging between 40 and $50 \mathrm{~nm}$ in diameter. Enhancement in the in-plane emission scattering is clearly visible only for the case when the film is composed of the random nanospheres. For ease of comparison, all plots share the same color scale. A: air, S: substrate, F: flat-film, C:Close-packed film, R: randomly-packed film.

From the combined spectrotemporal dynamics and electromagnetic simulations, we are able to build a mechanistic picture of how low-threshold random lasing occurs in these thin-films composed of nanospheres that individually display weak scattering properties. The sub- 
diffraction length scales serve to confine the incoming light fields within the bifunctional gain media and selectively outcompete extended modes that allow the emitted photons to diffuse throughout micron-thick samples ${ }^{13,17,26}$. In addition, the smaller nanospheres reduce the voids, i.e. non-radiative losses created by air pockets, by providing a closer packing capacity (greater gain-to-volume fraction). To estimate the magnitude of this effect, we can assume a certain packing arrangement (see Supplementary Information, Figure S6) and compute the gain-to-void fraction as the particle size is changed. We see that decreasing the nanospheres diameter from $200 \mathrm{~nm}$ to $35 \mathrm{~nm}$ results in a drastic decrease of the void feature size by about 90 percent. Consequently, this allows more emitted photons to interact with the gain medium containing excitons that have not recombined yet, increasing coherent scattering processes for light to be amplified, accelerating their decay via amplified stimulated emission.

In conclusion, we have demonstrated low-threshold excitonic UV-blue lasing $\left(<75 \mu \mathrm{J} / \mathrm{cm}^{2}\right)$ in an ultrathin-film of coupled nanospheres. Resolving the sub-ps absorption and emission dynamics below and above threshold provide insights into the material requirements for lasing in disordered materials. For example, we show that the gain mechanism is excitonic random lasing via multiple in-plane scattering, despite the use of weakly scattering $35 \mathrm{~nm}$ diameter nanostructures and sub-wavelength film thicknesses. Our results suggest that coupled, highly crystalline nanostructures, fabricated by low-cost solution processing methods, can function as viable building blocks for high-performance optoelectronic devices. 


\section{METHODS}

Fabrication of $\mathrm{ZnO}$ nanospheres sample. Hydrothermally grown $\mathrm{ZnO}$ nanostructures that are dispersed in ethanol with an average size of $\sim 35 \mathrm{~nm}$ and $40 \%$ weight concentration ( $\sim 10 \%$ volume concentration) was obtained from Sigma Aldrich. This solution was further diluted to $0.7 \%$ volume concentration and spun-cast at $10,000 \mathrm{rpm}$ for 60 seconds and baked at $300^{\circ} \mathrm{C}$ for 10 minutes to remove the residual ethanol solvent. To vary the sample thickness, different concentration of the dispersed solution was used. The resulting spin-coated nanostructures formed a close- packed thin film as shown in the scanning electron micrograph of Figure 1a. The thicknesses of the films were estimated using ellipsometric techniques and scanning electron microscopy ${ }^{41}$. As control, similar samples were annealed for a short duration using a rapid thermal process (RTP conditions: 5 minutes at $800^{\circ} \mathrm{C}$ ) and the dispersed solution was placed in a $1 \mathrm{~mm}$ cuvette for emission studies.

Optical characterization. For all optical spectroscopy measurements (steady-state emission and ultrafast transient and emission measurements), an excitation pulse of wavelength $280 \mathrm{~nm}$, pulse duration of $100 \mathrm{fs}$ and focus to a spot size of about $500 \mu \mathrm{m}$ in diameter was used (pump). The experiments were conducted using a Ti:Sapphire laser system (Spectra Physics), with the pump being generated using a commercial optical parametric amplifier (OPA, Light Conversion Ltd). For transient absorption measurements, a supercontinuum probe was generated by focusing the $800 \mathrm{~nm}$ fundamental light into a calcium fluoride plate. Shot-by-shot detection was achieved using fast silicon multichannel detectors (Ultrafast Systems LLC). For ultrafast transient emission experiments, a high sensitivity home-built setup based on the optical Kerr effect was used $^{25}$. The modularity of our setup allows low emissive states to be gated/probed using benzene as the Kerr medium ${ }^{22,42}$ in the low fluence limit, with a temporal resolution of $\sim 530 \mathrm{fs}$, and quartz in the high fluence limit, with a time resolution of $\sim 220$ fs. The gated emission signal is collimated and hereafter focused onto an imaging, nitrogen-cooled spectrometer (iHR320), equipped with a chargecoupled camera (SymphonyII, HORIBA Jobin Yvon). More details are also presented in the Supplementary Information. 
Electromagnetic Simulations. To confirm the effect of a random assembly of nanostructures on its inplane emission scattering, we use full-field 3D finite-difference time-domain software (Lumerical Solutions ${ }^{\circledR}$ ) to calculate the near-field electromagnetic distributions in a variety of films with a constant thickness of $140 \mathrm{~nm}$. The volume simulated was $500 \times 500 \times 700 \mathrm{~nm}^{3}$ with $\mathrm{x}$ and $\mathrm{y}$ having periodic boundary conditions while $\mathrm{z}$ had a perfect matching layer (parameters were 24 layers, kappa $=2$ and sigma $=0.25$ ). A set of dipole emitters (arranged to simulate random polarization and in the spectral range $350-450 \mathrm{~nm}$ ) was placed in the simulation volume while XZ-plane monitors were placed at the center of the simulated volume, i.e. $y=0$, to record the transmission and electromagnetic near-field distributions of the system. In all cases, these parameters were kept constant with only the film morphology being varied. Simulations were performed on (i) flat and unstructured, (ii) closed-packed nanospheres and (iii) randomly distributed nanospheres thin-films. For the random nanostructures, we use the uniform randomly distributed nanophere function with diameter parameter set to range around $40 \mathrm{~nm}$. The same $\mathrm{ZnO}$ dielectric value was used for all configurations. 


\section{Supporting Information}

Supporting information available:

\section{Corresponding Author}

Address correspondence to MYS at msfeir@bnl.gov.

\section{Author Contributions}

XL, VM, KA, and MYS designed the experiment. XL fabricated the samples. KA and XL collected and KA and MYS analyzed the ultrafast measurements. All authors interpreted the results and contributed to writing the manuscript.

\section{Funding Sources}

This research used resources of the Center for Functional Nanomaterials, which is a U.S. DOE Office of Science User Facility, at Brookhaven National Laboratory under Contract No. DESC0012704. Work at the College University of New York is supported by the National Science Foundation through Grant No. DMR 1410249.

\section{Notes}

The authors declare no competing financial interest.

\section{ACKNOWLEDGMENT}

The authors thank Erik Busby and Dmytro Nykypanchuk for helpful discussions. 


\section{References}

1. Dang, C.; Lee, J.; Breen, C.; Steckel, J. S.; Coe-Sullivan, S.; Nurmikko, A., Red, Green and Blue Lasing Enabled by Single-Exciton Gain in Colloidal Quantum Dot Films. Nature Nanotech. 2012, 7, 335339.

2. Feng, L.; Wong, Z. J.; Ma, R.-M.; Wang, Y.; Zhang, X., Single-Mode Laser by Parity-Time Symmetry Breaking. Science 2014, 346, 972-975.

3. Ma, R.-M.; Oulton, R. F.; Sorger, V. J.; Bartal, G.; Zhang, X., Room-Temperature SubDiffraction-Limited Plasmon Laser by Total Internal Reflection. Nature Mater. 2011, 10, 110-113.

4. Cooney, R. R.; Sewall, S. L.; Sagar, D. M.; Kambhampati, P., Gain Control in Semiconductor Quantum Dots Via State-Resolved Optical Pumping. Phys. Rev. Lett. 2009, 102, 127404.

5. Ivanov, S. A.; Achermann, M., Spectral and Dynamic Properties of Excitons and Biexcitons in Type-Ii Semiconductor Nanocrystals. ACS Nano 2010, 4, 5994-6000.

6. Sewall, S. L.; Franceschetti, A.; Cooney, R. R.; Zunger, A.; Kambhampati, P., Direct Observation of the Structure of Band-Edge Biexcitons in Colloidal Semiconductor Cdse Quantum Dots. Phys. Rev. B 2009, 80, 081310.

7. Cooney, R. R.; Sewall, S. L.; Sagar, D. M.; Kambhampati, P., State-Resolved Manipulations of Optical Gain in Semiconductor Quantum Dots: Size Universality, Gain Tailoring, and Surface Effects. $J$. Chem. Phys. 2009, 131, 164706.

8. Klimov, V. I.; Ivanov, S. A.; Nanda, J.; Achermann, M.; Bezel, I.; McGuire, J. A.; Piryatinski, A., Single-Exciton Optical Gain in Semiconductor Nanocrystals. Nature 2007, 447, 441-446.

9. Moreels, I.; Rainò, G.; Gomes, R.; Hens, Z.; Stöferle, T.; Mahrt, R. F., Nearly TemperatureIndependent Threshold for Amplified Spontaneous Emission in Colloidal Cdse/Cds Quantum Dot-inRods. Adv. Mater. 2012, 24, OP231-OP235.

10. Guzelturk, B.; Kelestemur, Y.; Olutas, M.; Delikanli, S.; Demir, H. V., Amplified Spontaneous Emission and Lasing in Colloidal Nanoplatelets. ACS Nano 2014, 8, 6599-6605.

11. Cirloganu, C. M.; Padilha, L. A.; Lin, Q.; Makarov, N. S.; Velizhanin, K. A.; Luo, H.; Robel, I.; Pietryga, J. M.; Klimov, V. I., Enhanced Carrier Multiplication in Engineered Quasi-Type-Ii Quantum Dots. Nat. Commun. 2014, 5.

12. Wang, J.; Genack, A. Z., Transport through Modes in Random Media. Nature 2011, 471, 345348.

13. Wiersma, D. S.; Lagendijk, A., Light Diffusion with Gain and Random Lasers. Phys. Rev. E 1996, 54, 4256-4265.

14. Cao, H.; Zhao, Y. G.; Ho, S. T.; Seelig, E. W.; Wang, Q. H.; Chang, R. P. H., Random Laser Action in Semiconductor Powder. Phys. Rev. Lett. 1999, 82, 2278-2281.

15. Klingshirn, C.; Fallert, J.; Zhou, H.; Sartor, J.; Thiele, C.; Maier-Flaig, F.; Schneider, D.; Kalt, H., 65 Years of Zno Research - Old and Very Recent Results. Phys. Status Solidi B 2010, 247, 14241447. 
16. Nakamura, T.; Firdaus, K.; Adachi, S., Electron-Hole Plasma Lasing in a Zno Random Laser. Phys. Rev. B 2012, 86, 205103.

17. Fallert, J.; Dietz, R. J. B.; Sartor, J.; Schneider, D.; Klingshirn, C.; Kalt, H., Co-Existence of Strongly and Weakly Localized Random Laser Modes. Nature Photon. 2009, 3, 279-282.

18. Toshihiro, N.; Hideki, F.; Ryo, N.; Keiji, S.; Yoshie, I.; Naoto, K.; Takeshi, T.; Sadao, A., Origins of Lasing Emission in a Resonance-Controlled Zno Random Laser. New J. Phys. 2014, 16, 093054 .

19. Apalkov, V. M.; Raikh, M. E.; Shapiro, B., Random Resonators and Prelocalized Modes in Disordered Dielectric Films. Phys. Rev. Lett. 2002, 89, 016802.

20. Fujiwara, H.; Hamabata, Y.; Sasaki, K., Numerical Analysis of Resonant and Lasing Properties at a Defect Region within a Random Structure. Opt. Express 2009, 17, 3970-3977.

21. Xing, G.; Xing, G.; Li, M.; Sie, E. J.; Wang, D.; Sulistio, A.; Ye, Q.-1.; Huan, C. H. A.; Wu, T.; Sum, T. C., Charge Transfer Dynamics in Cu-Doped Zno Nanowires. Appl. Phys. Lett. 2011, 98, 1021053.

22. Appavoo, K.; Liu, M.; Sfeir, M. Y., Role of Size and Defects in Ultrafast Broadband Emission Dynamics of Zno Nanostructures. Appl. Phys. Lett. 2014, 104, 133101.

23. Almand-Hunter, A. E.; Li, H.; Cundiff, S. T.; Mootz, M.; Kira, M.; Koch, S. W., Quantum Droplets of Electrons and Holes. Nature 2014, 506, 471-475.

24. Busby, E.; Thibert, A.; Fuzell, J.; Arrington, D. C.; Jawaid, A. M.; Snee, P. T.; Larsen, D. S., Ultrafast Exciton Dynamics in Colloidal Aluminum Phosphide Nanocrystals. Chem. Phys. Lett. 2013, $557,129-133$.

25. Appavoo, K.; Sfeir, M. Y., Enhanced Broadband Ultrafast Detection of Ultraviolet Emission Using Optical Kerr Gating. Rev. Sci. Instrum. 2014, 85, 055114.

26. Berger, G. A.; Kempe, M.; Genack, A. Z., Dynamics of Stimulated Emission from Random Media. Phys. Rev. E 1997, 56, 6118-6122.

27. Siegman, A. E., Lasers. University Science Books: Sausalito, CA, 1986.

28. Guillaume, C. B.; Debever, J.-M.; Salvan, F., Radiative Recombination in Highly Excited Cds. Phys. Rev. 1969, 177, 567-580.

29. Hönerlage, B.; Klingshirn, C.; Grun, J. B., Spontaneous Emission Due to Exciton-Electron Scattering in Semiconductors. Phys. Status Solidi B 1976, 78, 599-608.

30. Guillet, T.; Brimont, C.; Valvin, P.; Gil, B.; Bretagnon, T.; Médard, F.; Mihailovic, M.; ZúñigaPérez, J.; Leroux, M.; Semond, F., et al., Laser Emission with Excitonic Gain in a Zno Planar Microcavity. Appl. Phys. Lett. 2011, 98, -.

31. Mariano, A. Z.; Federico, C.; Sven, M.; Carsten, R., Optically Pumped Nanowire Lasers: Invited Review. Semicond. Sci. Technol. 2010, 25, 024001. 
32. Yamamoto, A.; Kido, T.; Goto, T.; Chen, Y.; Yao, T.; Kasuya, A., Dynamics of Photoexcited Carriers in Zno Epitaxial Thin Films. Appl. Phys. Lett. 1999, 75, 469-471.

33. Bauer, C.; Boschloo, G.; Mukhtar, E.; Hagfeldt, A., Ultrafast Relaxation Dynamics of Charge Carriers Relaxation in Zno Nanocrystalline Thin Films. Chem. Phys. Lett. 2004, 387, 176-181.

34. Mehl, B. P.; Kirschbrown, J. R.; Gabriel, M. M.; House, R. L.; Papanikolas, J. M., Pump-Probe Microscopy: Spatially Resolved Carrier Dynamics in Zno Rods and the Influence of Optical Cavity Resonator Modes. J. Phys. Chem. B 2012, 117, 4390-4398.

35. Toshine, Y.; Takeda, J.; Ko, H. J.; Yao, T., Conversion of an Electron-Hole Plasma into a High Density Excitonic State in Zno Epitaxial Thin Films. Phys. Status Solidi C 2004, 1, 839-842.

36. Haug, H.; Grob, K., Exciton Laser Theory. Phys. Lett. A 1967, 26, 41-42.

37. Haug, H., Theory of Laser Action Involving Free Excitons and Lo-Phonon-Assisted Transitions. J. Appl. Phys. 1968, 39, 4687-4695.

38. Hendry, E.; Koeberg, M.; Bonn, M., Exciton and Electron-Hole Plasma Formation Dynamics in Zno. Phys. Rev. B 2007, 76, 045214.

39. Versteegh, M. A. M.; Kuis, T.; Stoof, H. T. C.; Dijkhuis, J. I., Ultrafast Screening and Carrier Dynamics in Zno: Theory and Experiment. Phys. Rev. B 2011, 84, 035207.

40. Zhang, X. H.; Chua, S. J.; Yong, A. M.; Yang, H. Y.; Lau, S. P.; Yu, S. F.; Sun, X. W.; Miao, L.; Tanemura, M.; Tanemura, S., Exciton Radiative Lifetime in Zno Nanorods Fabricated by Vapor Phase Transport Method. Appl. Phys. Lett. 2007, 90, 013107.

41. Liu, X.; Goldberg, D.; Menon, V. M., Formation of Microcavity Polaritons in Zno Nanoparticles. Opt. Express 2013, 21, 20620-20625.

42. Appavoo, K.; Liu, M.; Black, C. T.; Sfeir, M. Y., Quantifying Bulk and Surface Recombination Processes in Nanostructured Water Splitting Photocatalysts Via in Situ Ultrafast Spectroscopy. Nano Lett. 2015, 15, 1076-1082. 
Table of Contents Figure -

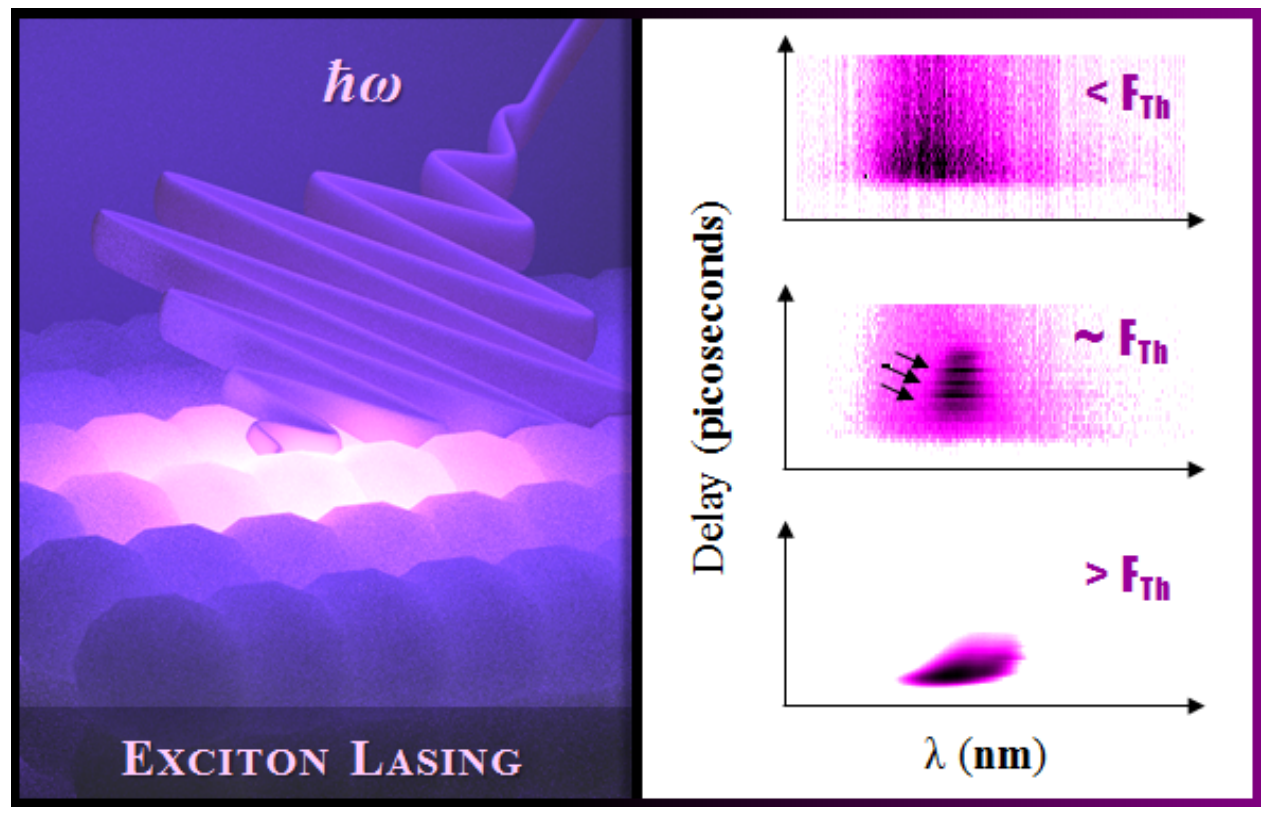

